\title{
4. Über die endlichen Gruppen der Algebrenklassen mit einem Zerfallungskörper.
}

\author{
Von Kenjiro Shoma.
}

(Eingegangen am 16. Februar, 1934)

Die vorliegende Arbeit schliesst sich an die Arbeit von R. Braner: Über die Konstruktion der Schiefkörper, die von endlichem Rang in bezug auf ein gegebenes Zentrum sind. $\left(^{1}\right)$ In dieser Arbeit hat R. Brauer das Faktorensystem eines einfachen hyperkomplexen Systems durch eine Erweiterung einer zyklischen Gruppe mit Hilfe der galoisschen Gruppe eines galoisschen Zerfällungskörpers charakterisiert. Er hat dann die Probleme über die Faktorensysteme auf gewisse Probleme in der Gruppentheorie, sowie auf gewisse Einbettungsprobleme in der Körpertheorie reduziert.

Wir betrachten im folgenden nicht nur ein einziges Faktorensystem sondern eine endliche Gruppe der Faktorensysteme, und wir beweisen die Sätze von R. Brauer für diesen allgemeineren Fall. Dabei legen wir aber die Darstellungen der einfachen Systeme durch verschränkte Produkte zugrunde und wir betrachten nur die darauftretenden Faktorensysteme.

\section{$\$ 1$. Charakterisierung einer endlichen Gruppe der Faktorensysteme durch eine abstrakte Gruppe. $\left({ }^{2}\right)$}

Es sei $K$ ein galoisscher Erweiterungskörper endlichen Grades eines vollkommenen Körpers $P$. Im folgenden betrachten wir nur einfache hyperkomplexe Systeme, die $P$ als Zentrum und $K$ als Zerfällungskörper besitzen. $\left({ }^{3}\right)$

Jedes System ist dann mit einem verschränkten Produkt

(1) R. Brauer, Journal für Math. 168 (1932), S. 44-64.

( ${ }^{2}$ Die hier anzugebende Konstruktion ist eine Analogon zur Schurschen Konstruktion der Darstellungsgruppe in der Theorie der Darstellungen einer endlichen Gruppe durch gebrochene lineare Substitutionen (Kollineationen). Vgl. I. Schur, Über die Darstellungen der endlichen Gruppen durch gebrochene lineare Substitutionen, Journal für Math. 127 (1904), S. 20-50. Untersuchungen über die Darstellungen der endlichen Gruppen durch gebrochene lineare Substitutionen, ebenda 132 (1907), S. 85-137.

Die folgende Beweisführung übernehme ich durch Modifizierung aus einem Asanoschen Beweis für die Existenz der Schurschen Darstellungsgruppe. Vgl. unsere gemeinsame Arbeit, Zur Theorie der Darstellungen einer endlichen Gruppe durch Kollineationen, die demnächst in Compositio Mathematica erscheinen wird.

( ${ }^{3}$ Z Zur folgenden Zusammenfassung vgl. R. Brauer, a. a. O. order H. Hasse, Theory of cyclic algebras over an algebraic number field, Kapitel 2, Transactions of the Amer. Math. Soc. 34 (1932), S. 171-214. 


$$
(c, K)=K u_{E}+K u_{s}+\ldots \ldots+K u_{T}
$$

ähnlich, wo $E, S, \ldots \ldots, T$ die Elemente der galoisschen Gruppe $(\mathfrak{S}$ von $K$ über $P$ bedeuten. Dabei gelten.

$$
a u_{S}=u_{S} a^{s}, \quad u_{S} u_{T}=c_{S, T} u_{S T}
$$

mit $a$ und $c_{s, r}$ aus $K$. Aus dem Assoziativgeset\% folgt bekanntlich

$$
c_{R, S} c_{R S, T}=c_{S, T}^{R^{-1}} c_{R, S T} .
$$

Zwei Faktorensysteme $\left\{c_{S, T}\right\},\left\{c_{S, T}^{\prime}\right\}$ heissen miteinander assoziiert, wenn es ein Elementensystem $n_{s}$ aus $K$ gibt, das der Bedingung

$$
c_{S, T}^{\prime}=\frac{n_{S} n_{T}^{s}}{n_{S T}} c_{S, T}
$$

genügt. Wir identifizieren im folgenden stets $u_{E}$ mit dem Einselement. Dann ist ersichtlich $c_{E, S}=c_{S, E}=1$ für jedes $S$ aus $(6$.

Die Gesamtheit der Klassen ähnlicher Systeme bildet durch direkte Multiplikation eine abelsche Gruppe, die wir die R. Brauersche Algebrenklassengruppe für $K$ nennen. Die Klassen assoziierter Faktorensysteme bilden durch die Multiplikationsregel $\left\{c_{S, r}\right\}\left\{c_{S, T}^{\prime}\right\}=\left\{c_{s, r} c_{S, T}^{\prime}\right\}$ eine zur Algebrenklassengruppe für $K$ isomorphe Gruppe.

Ist das Faktorensystem eines Systems $(c, K)$ mit einem aus Einheitswurzeln bestehenden Faktorensystem assoziiert, so sagen wir nach R. Brauer, dass $K$ ein regulärer Zerfällungskörper von $(c, K)$ ist. Die Gesamtheit der Klassen ähnlicher Systeme, die $K$ als einen regulären Zerfällungskörper besitzen, bildet eine endliche Untergruppe der Algebrenklassengruppe für $\boldsymbol{K}$. Die Untersuchung dieser Gruppe ist das Ziel der vorliegenden Arbeit.

Es sei $\Omega_{f}$ eine endliche Gruppe von Faktorensystemen, nicht von Klassen assoziierter Faktorensysteme. Die Ordnung von $\mathfrak{R}_{f}$ sei gleich $e$. Dann besteht jedes Faktorensystem aus $\mathfrak{R}_{f}$ aus Einheitswurzeln. Die Faktorensysteme, die eine Basis von $\mathfrak{K}_{\mathcal{T}}$ bilden, bezeichnen wir mit $\left\{c_{S, T}^{(1)}\right\}, \ldots \ldots$, $\left\{c_{s, r}^{(r)}\right\}$, wo die Ordnung von $\left\{c_{S, T}^{(i)}\right\}$ gleich $e_{i}$ ist, also ist $e=e_{1} e_{2} \ldots \ldots e_{r}$. Dann lässt sich jedes Faktorensystem $\left\{c_{S, r}\right\}$ durch

$$
\left.\left\{c_{S, T}\right\}=\left\{c_{S, T}^{(1)}\right\}^{c_{1}} \ldots \ldots\left\{c_{S, T}^{(r)}\right\}\right\}^{r_{r}}
$$

darstellen. Das Faktorensystem $\left\{c_{s, r}^{(i)}\right\}$ kamn man durch

$$
c_{S, T}^{(i)}=\rho_{i}^{a_{S, T}^{(i)}}
$$

mit einem ganzen $a_{s, r}^{(i)}$ ausdrücken, wo $\rho_{i}$ eine beliebige aber feste primitive $e_{i}$-te Einheitswurzel bedeutet. Da die Ordnung von $\left\{c_{S, r}^{(i)}\right\}$ gleich $e_{i}$ ist, so ist der grösste gemeinsame 'Teiler' von $a_{s, r}^{(i)}$ für alle $S, T$ aus (f) zu $e_{i}$ teilerfremd. Dann ist 


$$
c_{s, r}=\prod_{i=1}^{r} \rho_{i}{ }^{c_{\ell} a_{S, r}^{(i)}}, 1 \leqq c_{i} \leqq e_{i} .
$$

Aus dem Assoziativgesetz (3) folgt nach (6)

$$
a_{R, S}^{(i)}+a_{R S, T}^{(i)} \equiv \alpha_{R^{-1}} a_{S, T}^{(i)}+a_{R, S T}^{(i)}
$$

wenn

$$
\boldsymbol{\rho}_{i}^{R^{-1}}=\boldsymbol{\rho}_{i}^{\boldsymbol{\alpha}_{R^{-1}}}
$$

ist. Bedeutet $\varepsilon$ eine primitive $m$-te Einheitswurzel, wo $m$ das kleinste gemeinsame Vielfache von $e_{1}, e_{2}, \ldots \ldots, e_{r}$ ist, so ist $\varepsilon$ natürlich in $K$ enthalten. Für jedes $S$ aus (i) ist $\varepsilon^{s}$ eine Potenz von $\varepsilon$. Ist $\varepsilon^{s}=\varepsilon^{\alpha}$, so ist $\rho_{i}^{S}=\rho_{i}^{\boldsymbol{\alpha}}$, da jedes $\rho_{i}$ eine Potenz voi $\varepsilon$ ist. Daher ist der Exponent $\alpha_{s}$ unabhängig vom Index $i$.

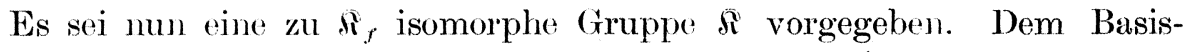
element $\left\{c_{s, r}^{(i)}\right\}$ entspreche dabei $C_{i}$ aus $\mathfrak{R}$. Wenn man

$$
C_{S, T}=\prod_{i=1}^{r} C_{i}^{a_{S, r}^{(i)}}
$$

setzt, so ist wegen (8)

$$
C_{R, S} C_{R S, t}=C_{S, r}^{a} C_{R, S T}^{-1}
$$

Wir führen nun neue Elemente $Z_{E}, Z_{S}, \ldots \ldots, Z_{r}$ ein, die den Elementen von (S) entsprechen, und wir setzen fest

$$
\begin{aligned}
Z_{S} Z_{r} & =C_{S, r} Z_{S r} \\
C Z_{S} & =Z_{S} C^{\alpha_{S}}
\end{aligned}
$$

für jedes $C$ ans $h$. Wir setzen ferner das Assoziativgesetz zwischen $C$ und $Z_{S}$ voraus. Dann bilden die Elemente $C Z_{S}$ eine Gruppe, wenn $C$ bzw. $S$ alle Elemente von $\Re$ bzw. $(\mathfrak{S}$ durchläuft. Zum Beweis zeigen wir, dass die Menge die Gruppenpostulate bestätigt. Dabei kann man ersichtlich $Z_{E}$ mit dem Einselement von $\AA$ identifizieren. Nach (11) und (12) ist das Produkt zweier Elemente der Menge eindeutig als ein Element der Menge bestimmt. Nach (12) ist $Z_{S^{-1}} C_{S, S^{-1}}^{-1}$ das reziproke Element von $Z_{S}$. Das Assoziativgesetz besteht in $\mathfrak{R}$, sowie zwischen $C$ und $Z_{s}$. Das Assoziativgesetz zwischen den $Z_{S}$ folgt aus (10) und (12). Wenn man das Element $Z_{S}^{-1} C Z_{S}$ wie üblich mit $C^{s}$ bezeichnet, so kann man (12) in der Form

$$
C^{s}=C^{\boldsymbol{\alpha}_{s}}
$$

darstellen. Die oben konstruierte Gruppe bezeichnen wir mit $\mathfrak{H}$. Dann ist $\mathfrak{5}$ eine Erweiterung von $\mathfrak{R}$ mit Hilfe von $(\mathfrak{S}$ im Schreierschen Sinne.

Durch Übergang zu einfachen Charakteren von $\Re$ erhält man aus $C_{S, r}$ die sämtlichen Faktorensysteme in $K_{f}$. Bedentet nämlich $\chi_{i}$ ein Charakter mit der Bedingung 
(13)

$$
\chi_{i}(C)=\chi_{i}\left(C_{i}\right)^{c_{i}}=\rho_{i}^{c_{i}} \quad \text { für } \quad Q=C_{1}^{c_{1}} C_{2}^{c_{2}} \ldots \ldots C_{r}^{c_{r}},
$$

wo $\rho_{i}$ wieder eine primitive $e_{i}$-te Einheitswurzel bedeutet, so lässt sich jeder Charakter eindeutig durch

$$
\chi(C)=\prod_{i=1}^{r} \chi_{i}(C)^{x_{i}}, \quad 1 \leqq x_{i} \leqq c_{i},
$$

darstellen. Nach (7) ist dann wegen (9) und (13)

$$
c_{S, T}=\prod_{i=1}^{r} \rho_{i}^{c_{i} a_{N, T}^{(i)}}=\prod_{i=1}^{r} \chi_{i}\left(C_{i}^{a_{S, T}^{(i)}}\right)^{c_{i}}=\prod_{i=1}^{r} \chi_{i}\left(C_{S, T}\right)^{c^{\prime} .}
$$

Dies besagt nichts anderes als, dass es einen Charakter $\chi$ gibt, der der Gleichung

$$
c_{S, T}=\chi\left(C_{S, r}\right)
$$

genügt. Da die Anzahl der Charaktere gleich der Ordnung von $\mathfrak{A}$ ist, so bilden die $\chi_{\left(C_{S, T}\right)}$ für jeden Charakter ein Faktorensystem aus $\mathfrak{R}_{f}$.

Zusammenfassend erhält man also:

Durch eine endliche Gruppe $\mathfrak{R}_{f}$ der Faktorensysteme wird eine Erveiterung einer zu $\mathfrak{R}_{f}$ isomorphen Gruppe $\mathfrak{A}$ mit Hilfe der galoisschen Gruppe (5) bestimmt. Dabei ist $C^{S}=C^{\alpha}$ s für jedes Element $C$ aus $\mathfrak{K}$ bzw. $S$ aus (\$), wenn $\rho^{s}=\rho^{\alpha}$ s fïr jede im Faktorensystern vorkommende Einheitswurzel ist. Dann

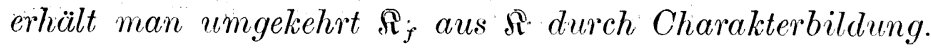

Der Körper $K$ enthalte nun eine primitive $m$-te Einheitswurzel $\rho$. Es sei eine Erweiterung $\mathfrak{H}$ einer abelschen Gruppe $\mathfrak{A}$ mit Hilfe von $(\mathfrak{S}$ derart vorgegeben, dass $A^{m}=E$ für jedes $A$ aus $\mathfrak{A}$, und dass $A^{s}=A^{\alpha_{s}}$ ist, falls $\rho^{s}=\rho^{\alpha}$ ist. Sind nun $Z_{E}, Z_{S}, \ldots \ldots, Z_{t^{\prime}}\left(Z_{E}=E\right)$ Repräsentanten der Restklassen von $\mathfrak{H}$ modulo $\mathfrak{A}$, so ist ersichtlich $Z_{\mathbb{S}} Z_{T} Z_{S T}^{-1}$ in $\mathfrak{A}$ enthalten. Setzt man

$$
Z_{S} Z_{T} Z_{S T}^{-1}=C_{S, T}
$$

so genügt das System von $C_{S, T}$ nach dem Assoziativgesetz von $\mathfrak{g}$ der Gleichung

Da jetzt

$$
C_{R, S} C_{R S, T}=C_{s, T}^{R^{-1}} C_{R, S T}=C_{S, T}^{\alpha_{R}-1} C_{R, S T}
$$

$$
\chi\left(C^{s}\right)=\chi(C)^{s}=\chi(C)^{\alpha_{s}}
$$

für jedes Element $C$ aus $\mathfrak{A}$ und für jeden Charakter $\chi$ ist, so genügen die $\chi\left(C_{S, t}\right)$ der Bedingung (3), also bilden sic ein Faktorensystem, da sie alle in $K$ liegen. Die Gesamtheit solcher Charaktere bildet eine Gruppe, die der durch $C_{s, t}$ erzeugten Untergruppe von $\mathfrak{A}$ isomorph ist.

\section{\$2. Zuordnung zwischen $\mathfrak{\Re}_{f}$ und $\mathfrak{A}$.}

Eine Untergruppe $\mathfrak{I}_{f}{ }^{\prime}$ von $\mathfrak{i}_{f}$ wird durch eine Untergruppe $\mathfrak{K}_{x}{ }^{\prime}$ der




der Bedingung $\chi(C)=1$ für jeden Charakter aus $\mathfrak{K}_{\chi}{ }^{\prime}$ genügen, bilden eine Untergruppe $\mathscr{R}^{\prime}$ von $\mathscr{R}$. Dann ist es bekannt, dass $\mathfrak{R}_{\chi}^{\prime}$ gerade die Charaktergruppe von $\mathfrak{R} / \mathscr{R}^{\prime}$ ist.

Umgekehrt sei eine Untergruppe $\mathfrak{I}^{\prime}$ von $\mathfrak{R}$ vorgegeben. Diejenigen Charaktere von $\mathfrak{R}$, die zugleich Charaktere der Faktorgruppe $\mathfrak{A} / \mathfrak{K}^{\prime}$ sind, bilden eine Untergruppe $\mathfrak{R}_{x}{ }^{\prime}$ der Charaktergruppe $\mathfrak{K}_{x}$. Dann ist ersichtlich $\mathfrak{R}_{x}{ }^{\prime}$ die Charaktergruppe von $\mathfrak{R} / \mathfrak{R}^{\prime}$. Damit ist eine bekannte eineindeutige Zuordnung zwischen den Untergruppen der Charaktergruppe $\mathfrak{R}_{x}$ und den Faktorgruppen der Gruppe $\mathfrak{A}$, also folglich eine eineindeutige Zuordnung zwischen den Untergruppen der Gruppe $\mathfrak{R}_{f}$ der Faktorensysteme und den Faktorgruppen der Gruppe $\Re$ aufgestellt.

Eine Untergruppe von $\mathfrak{R}$ ist stets ein Normalteiler von $\mathfrak{j}$. venn transformiert man ein Element $A$ aus $\Omega$ mit $Z_{s}$, so erhält man $A^{s}=A^{\alpha_{s}}$; Mit $A$ ist daher auch $A^{S}$ für jedes $S$ aus (is in einer Untergruppe von $\mathfrak{K}$ enthalten. Daher kann man nach oben eine eineindeutige Zuordnung zwischen den Untergruppen $\mathfrak{K}_{f}^{\prime}$ von $\mathfrak{K}_{f}$ und den Faktorgruppen $\mathfrak{n} / \mathfrak{K}^{\prime}$ modulo einem in $\mathfrak{R}$ enthaltenen Normalteiler $\mathfrak{K}^{\prime}$ aufstellen, so dass $\mathfrak{R}_{f}^{\prime}$ aus den sämtlichen Faktorensystemen besteht, die durch die Charaktere von $\mathscr{R} / \mathscr{K}^{\prime}$ ermittelt werden.

\section{\$3. Reduzierte Erweiterungsgruppe $\mathfrak{H}$.}

Besitzt ந) eine Untergruppe $\mathfrak{Y}^{\prime}$ mit der Eigenschaft $\mathfrak{S}=\mathfrak{S}^{\prime} \mathfrak{\mathfrak { A }}$, so folgt aus dem Isomorphismus von $(\mathfrak{S}$ auf $\mathfrak{\mathfrak { H }} / \mathfrak{K}$ nach dem zweiten Isomorphiesatz der Isomorphismus von (G) auf $\left.\mathfrak{H}^{\prime} /[\mathfrak{H}), \mathfrak{X}\right]$. Ist nämlich

$$
\mathfrak{n}=\Re U_{E}+\Re U_{S}+\ldots \ldots+\Re U_{T}
$$

die Nebengruppenzerlegung von $\mathfrak{g}$ modulo $\mathfrak{K}$, so hat die Nebengruppenzerlegung von $\mathfrak{h}^{\prime}$ modulo $\mathfrak{R}^{\prime}=\left[\mathfrak{h}^{\prime}, \mathfrak{R}\right]$ die Gestalt

$$
\mathfrak{h}^{\prime}=\mathfrak{R}^{\prime} U_{t^{\prime}}^{\prime}+\mathfrak{\Re}^{\prime} U_{s}^{\prime}+\ldots \ldots+\Re^{\prime} U_{T}^{\prime},
$$

wobei $\mathfrak{K}^{\prime} U_{\mathrm{s}}^{\prime}$ in $\mathfrak{K} U_{\mathrm{s}}$ enthalten ist, also ist ersichtlich

$$
U_{s}^{\prime}=N_{S} U_{s}
$$

mit einem Element $N_{s}$ aus $\mathfrak{R}$. Es ist damn

$$
U_{S}^{\prime} U_{T}^{\prime}=C_{S, r}^{\prime} U_{S T}^{\prime}, \quad C_{S, T}^{\prime}=N_{S} N_{T}^{s} N_{S T}^{-1} C_{\mathrm{s}, T} .
$$

Durch Übergang zum Charakter von $\mathfrak{A}$ ergibt sich hieraus wegen (15)

$$
\chi\left(C_{S, r}^{\prime}\right)=\frac{\chi\left(N_{S}\right) \chi\left(N_{r}\right)^{s}}{\chi\left(N_{S T}\right)} \chi\left(C_{S, r}\right)
$$

Ein aus $m$-ten Einheitswurzeln bestehendes Faktorensystem $\left\{c_{S, T}\right\}$ heisst einem Faktorensystem $\left\{c_{s, r}^{\prime}\right\}$ eng assoziiert, wenn die Gleichungen 


$$
c_{S, T}^{\prime}=\frac{n_{S} n_{T}^{s}}{n_{S T}} c_{S, r}
$$

durch $m$-te Einheitswurzeln $n_{S}$ lösbar sind. Damn besagt (16), dass das Faktorensystem $c_{S, T}=\chi\left(C_{S, r}^{\prime}\right)$ zum $c_{S, r}^{\prime}=\chi\left(C_{S, r}^{\prime}\right)$ eng assoziiert ist. Damit ist also gezeigt: Jedes durch einen Charakter von $\mathfrak{i}$ definierte Faktorensystem ist einem durch einen Charakter von $\mathfrak{K}^{\prime}$ definierten Faktorensystem eng assoziiert. Bezeichnet man nun die nach $\$ 2 \mathrm{zu} \mathfrak{R} / \mathfrak{K}^{\prime}$ entsprechende Untergruppe von $\mathfrak{R}_{f}$ mit $\mathfrak{K}_{f}^{\prime}$, so ist das Faktorensystem aus $\mathfrak{K}_{f}^{\prime}$ zum Einssystem eng assoziiert. Je zwei Faktorensysteme aus einer selben Nebengruppe modulo $\mathfrak{R}_{f}^{\prime}$ sind zueinander eng assoziiert.

Sind nun alle Faktorensysteme aus $\mathfrak{X}_{f}$ zum Einssystem eng assoziiert, so ist $\mathfrak{H}$ das Produkt von $\mathfrak{R}$ und einer zu ('5) isomorphen Gruppe (\$S'. Es sei nämlich in (15)

$$
\chi_{i}\left(C_{S, T}\right)=\frac{\omega_{N} \omega_{T}^{S}}{\omega_{S T}}
$$

wo $\omega_{S}$ eine $e_{i}$-te Einheitswurzel bedeutet. Dam kann man eine Potenz $N_{s}^{(i)}$ von $C_{i}$ so annehmen, dass

$$
\omega_{s}=\chi_{i}\left(N_{s}^{(i)}\right)
$$

gilt. Setzt man nun

so ist

$$
N_{s}=N_{S}^{(1)} N_{S}^{(2)} \ldots \ldots N_{S}^{(r)},
$$

$$
c_{S, T}=\prod_{i=1}^{r} \chi_{i}\left(C_{S, T}\right)^{c_{i}}=\prod_{i=1}^{r} \frac{\chi_{i}\left(N_{S}\right)^{c i} \chi_{i}\left(N_{T}\right)^{c_{i} S}}{\chi_{i}\left(N_{S T}\right)^{c_{i}}}
$$

Also ist

$$
\chi\left(C_{S, r}\right)=\chi\left(N_{S} N_{T}^{s} N_{s T}^{-1}\right)
$$

für jeden Charakter $\chi$ und folglich

$$
C_{S, r}=N_{s} N_{r}^{s} N_{s t}^{-1} \text {. }
$$

Daher bilden die $N_{S}^{-1} Z_{S}$ eine $z u$ (5s isomorphe Gruppe (5') und ersichtlich $\mathfrak{H}=\left(\mathfrak{S}^{\prime} \mathfrak{R}\right.$.

Es sei nun $\mathfrak{R}_{x}{ }^{\prime}$ eine Untergruppe von $\mathfrak{I}_{x}$ derart, dass $\left\{\chi\left(C_{S, r}\right)\right\}$ für $\chi$ aus $\mathfrak{R}_{x}{ }^{\prime}$ zum Einssystem eng assoziiert ist. Bezeichnet man die zu $\mathfrak{R}_{x}{ }^{\prime}$ entsprechende Faktorgruppe mit $\mathfrak{R} / \mathfrak{K}^{\prime}$, so ist $\mathfrak{R}_{x}{ }^{\prime}$ die Charaktergruppe von $\mathfrak{N} / \mathfrak{N}^{\prime}$. Durch die Erweiterungsgruppe von $\mathfrak{K} / \mathfrak{R}^{\prime}$ mit Hilfe von $(\mathfrak{S}$, d.h. durch die Gruppe $\mathfrak{H} / \mathfrak{\Re}^{\prime}$ wird dann die Faktorensysteme $\chi\left(C_{S, T}\right)$ mit $\chi$ aus $\mathfrak{A}_{\chi}^{\prime}$ 'definiert, die nach der Voraussetzung sämtlich zum Einssystem assoziiert sind. Daher ist $\mathfrak{H} / \mathfrak{\Re}^{\prime}$ das Produkt einer zu $\left(\mathfrak{S}\right.$ isomorphen Gruppe $\mathfrak{S}^{\prime} / \mathfrak{\Re}^{\prime}$ und $\mathfrak{K} / \mathfrak{K}^{\prime}$. Die Gruppe $\mathfrak{H}^{\prime}$. ist dann eine Erweiterung von $\mathfrak{\Omega}^{\prime}$ mit Hilfe von $(\mathfrak{5}$. Es ist jetzt ersichtlich, dass $\mathfrak{H}=\mathfrak{H}^{\prime} \mathfrak{\Re}$ ist. 
Eine Erweiterung $\mathfrak{H}$ heisst reduziert, wenn es keine echte Untergruppe $\mathfrak{\mathfrak { g }}^{\prime}$ mit der Eigenschaft $\mathfrak{H}=\mathfrak{H}^{\prime} \mathfrak{R}$ gibt. Ist $\mathfrak{H}$ eine reduzierte Erweiterung, so besitzt die dadurch definierte Gruppe der Faktorensysteme keine echte Untergruppe, die aus den zum Einssystem eng assoziierten Faktorensystemen besteht.

\section{\$4. Erweiterung des Zerfällungskörpers $K$.}

Wir untersuchen jetzt, wie sich die Gruppe $\mathfrak{H}$ ändert, wenn man statt $K$ einen $K$ umfassenden galoisschen Erweiterungskörper $K^{*}$ endlichen Grades über $P$ betrachtet. Es sei $\left(5^{*}\right.$ die galoissche Gruppe von $K^{*}$ über $P, \&$ die Invariatengruppe für $K$. Dann ist $(6)^{*} / \mathbb{Q}$ bekanntlich $z u$ (\$) isomorph. Ist ferner

$$
6^{*}=2 Z_{E}+2 Z_{S}+\ldots \ldots+2 Z_{T}
$$

die Nebengruppenzerlegung von $\left(^{*}\right.$ modulo dem Normalteiler $\mathfrak{R}$, wo $\mathbb{8} Z_{S}$ die deni Element $S$ aus $(5)$ entsprechenden Nebengruppe bedeutet, so ist es nach R. Brauer bekannt, dass zwei verschränkte Produkte $\left(c^{*}, K^{*}\right)$ und $(c, K)$ ähnlich sind, wenn

$$
r_{A, B}^{*}=c_{s, r} \text { für } \quad A \equiv Z_{S}, \quad B \equiv Z_{r} \quad(\bmod \mathfrak{l})
$$

ist. Dann heisst das Faktorensystem $\left\{c_{A, B}^{*}\right\}$ eine Erweiterung des Faktorensystems $\left\{c_{s, r}\right\}$. Durch diese Erweiterung erhält man aus $\mathfrak{S}$ eine Gruppe $\mathfrak{H}^{*}$, die eine Erweiterung von $\mathfrak{R}$ mit Hilfe von ( $\mathfrak{S}^{*}$ ist. Man erkennt sofort, dass $\mathfrak{H}^{*}$ durch $Z_{A}^{\prime}$ ( $A$ aus $\left(5^{*}\right)$ und $C_{S, T}$ mit den folgenden definierenden Relationen

$$
\begin{aligned}
& Z_{A}{ }^{\prime} Z_{B}{ }^{\prime}=C_{S, r} Z_{A B}^{\prime} \quad \text { für } \quad A \equiv Z_{s}, \quad B \equiv Z_{T} \quad(\bmod \mathfrak{I}) \\
& C Z_{A}{ }^{\prime}=Z_{A}{ }^{\prime} C^{\alpha}
\end{aligned}
$$

erzeugt. Dafür hat man natürlich auch die definierenden Relationen für $C_{S, r}$ hinzufügen. Dann ist $\mathfrak{Q}$ ersichtlich ein mit $\mathfrak{A}$ elementfremder Normalteiler von $\mathfrak{H}^{*}$.

Ist umgekehrt ein verschränktes Produkt $\left(c^{*}, K^{*}\right)$ vorgegeben, dessen Faktorensystem in einem in $K^{*}$ enthaltenen galoisschen Erweiterungskörper $K$ von $P$ liegt und der Bedingung

$$
c_{A, B}^{*}=c_{A^{\prime}, B^{\prime}}^{*} \quad \text { für } \quad A \equiv A^{\prime}, \quad B \equiv B^{\prime} \quad(\bmod \mathfrak{Z})
$$

genügt, wo $\mathfrak{Q}$ wie oben die Invariantengruppe für $K$ bedeutet, so kann man ein Faktorensystem von $(c, K)$ so wählen, dass das Faktorensystem $\left\{c_{A, B}^{*}\right\}$ eine Erweiterung des Faktorensystems $\left\{c_{S, T}\right\}$ ist.

Wenn man diese Tatsache auf $\mathfrak{H}^{*}$ überträgt, so erhält man : Aus (19) folgt (18) bei passender Wahl der Elemente $C_{S, T}$. Die Bedingung (18) ist aber damit äquivalent, dass $\mathfrak{Q}$ ein mit $\mathfrak{A}$ elementfremder Normalteiler von $\mathfrak{H}^{*}$ sein kann. Denn aus (18) folgt, dass $\mathfrak{R}$, wie oben schon bemerkt, ein 
mit $\mathfrak{A}$ elementfremder Normalteiler von $\mathfrak{S}^{*}$ ist. Umgekehrt sei $\mathbb{Q}$ ein mit $\mathfrak{A}$ elementfremder Normalteiler von $\mathfrak{H}$. Dann ist das Produkt $\mathfrak{R} \mathfrak{A}$ direkt. Daher ist

$$
\begin{aligned}
& Z_{A}{ }^{\prime} C^{\alpha} s=C Z_{A}{ }^{\prime} \quad \text { für } A \equiv Z_{s} \quad(\bmod \mathfrak{R}) \text {, } \\
& Z_{A}{ }^{\prime} Z_{P}{ }^{\prime} Z_{B}{ }^{\prime} Z_{Q}{ }^{\prime}=Z_{A}{ }^{\prime} Z_{B}^{\prime}{ }^{P} Z_{P}{ }^{\prime} Z_{Q}{ }^{\prime}=Z_{A B}^{\prime}{ }^{P} C_{P, Q} Z_{P Q}^{\prime}=C_{P, Q} Z_{{ }_{1 B}{ }^{P}}^{\prime} Z_{P Q}^{\prime}=C_{P, Q} Z_{A P Q}^{\prime}, \\
& Z_{A}{ }^{\prime} Z_{P}{ }^{\prime} Z_{B}{ }^{\prime} Z_{Q}{ }^{\prime}=Z_{A P}{ }^{\prime} Z_{B Q}^{\prime}=C_{A P, B Q} Z_{A P, B Q}^{\prime} \quad \text { für } A, B \text { aus } \& \text {. }
\end{aligned}
$$

Daher ist

$$
C_{A P, B Q}=C_{P, Q} \quad \text { für } A, B \text { aus } ? .
$$

Zusammenfassend erhält man also:

Beim. Übergang von $K$ zu $K^{*}$ erlü̈lt man aus $\mathfrak{S}$ eine Erweiterung $\mathfrak{j}^{*}$ von $\AA$ mit Hilfe der galoisschen Gruppe $\mathfrak{6}^{*}$ von $K^{*}$, die einen zur Invariantenginppe von $K$ isomorphen mit $\mathfrak{\AA}$ elementfremden Normalteiler besitzt. Besitzt umgekehrt $\mathfrak{H}^{*}$ einen mit $\mathfrak{A}$ elementfremden Normalteiler $\mathbb{\&}$ und liegen die Charaktere von $\mathfrak{R}$ sämtlich in einem Unterkörper $K$ mit der zu $\mathfrak{Z}$ isomorphen Invariantengruppe, so ist der Körper $K$ auch ein galoisscher Zerfüllungskörper. Beim Übergang von $K^{*}$ zu $K$ erhält man aus ந* eine Erweiterung நi von $\mathfrak{\AA}$ mit Hilfe der galoisschen Gruppe $(5$ von $K$.

\section{\$5. Bedingung für die Ähnlichkeit.}

Ist $\mathfrak{H}$ eine reduzierte Erweiterung von $\mathfrak{A}$ mit Hilfe von $(\mathfrak{B}$, so ist die Faktorgruppe $\mathfrak{H} / \mathfrak{R}^{\prime}$ für jede Untergruppe $\mathfrak{R}^{\prime}$ von $\mathfrak{R}$ eine reduzierte Erweiter-


$\mathfrak{h} / \mathscr{R}^{\prime}=\mathfrak{H}^{\prime} \mathfrak{K} / \mathfrak{R}^{\prime}$, also ist $\mathfrak{H}^{\prime} \mathfrak{\Re}=\mathfrak{H}$. Da aber nach der Voraussetzung $\mathfrak{g}$ reduziert ist, so ist $\mathfrak{J}^{\prime}=\mathfrak{H}$, d.h. $\mathfrak{H} / \mathfrak{R}^{\prime}$ ist reduziert.

Falls $\mathfrak{K}$ zyklisch ist, sind die durch $\mathfrak{h}$ definierten Faktorensysteme nach R. Brauer a.a.O. dann und nur dann zum Einssystem assoziiert, wenn man $K$ in einem galoisschen Erweiterungskörper $K^{*}$ mit der zu $\mathfrak{g}$ isomorphen galoisschen Gruppe einbetten kann.

Ist also im allgemeinen $K$ in einem galoisschen Erweiterungskörper $K^{*}$ mit der zu $\mathfrak{H}$ isomorphen galoisschen Gruppe über $P$ eingebettet, so ist jedes Faktorensystem, das durch $\mathfrak{h}$ definiert wird, zum Einssystem assoziiert. Es sei num

$$
\mathfrak{R}=\mathfrak{R}_{1} \times \mathfrak{R}_{2} \times \ldots \ldots \times \mathfrak{R}_{r}
$$

und $\mathfrak{R}_{i}$ zyklisch. Die Faktorgruppe $\mathfrak{H} / \mathfrak{R}_{1} \times \ldots \ldots \times \mathfrak{R}_{i-1} \times \mathfrak{R}_{i+1} \ldots \ldots \times \mathfrak{N}_{\text {. }}$. bezeichnen, wir mit $\mathfrak{H}_{i}$, die einer Erweiterung von $\mathfrak{R}_{i}$ mit Hilfe von $(5)$ isomorph ist. Dann ist die obige Tatsache mit der folgenden äquivalent: Ist $K$ in einem galoisschen Erweiterungskörper $K_{i}$ mit der zu $\tilde{\mathfrak{j}}_{i}$ isomorphen galoisschen Gruppe über $P$ eingebettet und ist der Durchschnitt

$$
\left[K_{i}, K_{1} K_{2} \ldots K_{i-1} K_{i+1} \ldots \ldots K_{r}\right]=K
$$


für jedes $i$, so ist jedes durch $\mathfrak{n}$ definierte Faktorensystem zum Einssystem assoziiert.

Wir beweisen num den Umkehrungssatz unter der Voraussetzung, dass $P$ ein algebraischer Zahlkörper endlichen Grades ist. Wir betrachten zunächst den Fall, dass $\mathfrak{R}$ zyklisch ist. Die Ordnung von $\mathfrak{R}$ sei gleich $e$. Ist nun ein Faktorensystem $\left\{c_{s, r}\right\}$, das durch $\mathfrak{h}$ definiert wird, zum Einssystem assoziiert:

$$
c_{s, r}=\frac{n_{s} n_{r}^{s}}{n_{s T}},
$$

so folgt hicraus

$$
n_{S}^{e} n_{T}^{e S}=n_{S T}^{e} .
$$

Es gibt also bekanntlich nach A. Speiser eine Zahl $\omega$ aus $K$ mit

$$
n_{S}^{e}=\omega^{1-s} \text {. }
$$

Dabei wird $\omega$ bis auf einen Faktor aus dem Grunkörper $P$ eindeutig bestimmt. Dann ist der Körper $K(\sqrt[e]{\omega})$ galoissch über $P$, da $\sqrt[e]{\omega^{s}}$ nach oben auch in $K(\mathfrak{j} \omega)$ liegt. Ist $\mathfrak{H}$ eine reduzierte Erweiterung von $\Re$ mit Hilfe von ( $\mathfrak{k}$, so ist terner nach R. Brauer a.a.O. der Grad von $K(\sqrt[p]{\omega})$ über $K$ gleich e und die galoissche Gruppe von $K(\stackrel{\mathfrak{V}}{\boldsymbol{\omega}})$ über $P$ zu $\mathfrak{j}$ isomorph.

Für einen beliebig vorgegebenen Erweiterungskörper $K^{*}$ endlichen Grades über $K$ kamn man sicher $\omega$ so annehmen, dass der Durchschnitt von $K(\sqrt[p]{\omega})$ und $K^{*}$ gleich $K$ wird. Dies gilt ferner für unendlich viele Zahlen $\omega$.

Betrachtet man nuimmehr den allgemeinen Fall, class $\mathfrak{i}$ nicht notwendig zyklisch ist, so wird jedes Faktorensystem durch eine Faktorgruppe von $\mathfrak{n}$ modulo einer Untergruppe von $\mathfrak{K}$ definiert, die auch reduziert ist. Nach dem oben gesagten kann man $K_{1}, K_{2}, \ldots \ldots, K_{r}$ so annehmen, dass (20) gelten für jedes $i$. Damit ist also gezeigt:

Die durch $\mathfrak{H}$ definierte Faktorensysteme sind, falls $\mathfrak{H}$ eine reduzierte Erweiterung von $\mathfrak{R}$ mit Hilfe von $(5)$ ist, dann und nur dann sümtlich, zum Einssystem assoziiert, wenn man $K$ in einem galoisschen Erweiterungskörper $K^{*}$ von $P$ mit der zu $\mathfrak{5}$ isomorphen galoisschen Gruppe einbetten kann.

Die Gesamtheit der zum Einssystem assoziierten Faktorensysteme in $\mathfrak{K}_{f}$ bildet eine Untergruppe $\mathfrak{R}_{f}^{\prime}$ von $\mathfrak{K}_{f}$. Dann wird $\mathfrak{K}^{\prime}$ nach $\$ 2$ dureh eine Faktorgruppe $\mathfrak{H} / \mathscr{\Re}^{\prime}$ definiert. Diese Faktorgruppe kann man daduch charakterisieren, dass man $K$ in einem $K^{*}$ mit der zu $\mathfrak{\mathfrak { H }} / \mathfrak{R}^{\prime}$ isomorphen galoisschen Gruppe einbetten kann. Die Gruppe $\boldsymbol{\Omega}^{\prime}$ ist dann eine minimale solche Untergruppe. Die Faktorgruppe $\mathfrak{R}_{f} / \mathfrak{R}_{f}^{\prime}$ ist der Gruppe der ähnlichen Klassen, also einer endlichen Untergruppe der Algebrenklassengruppe isomorph. 
$\mathfrak{R}_{f}^{\prime}$ ist aber die Untergruppe der Gruppe $\mathfrak{R}_{f}$ der Faktorensysteme, die durch eine Untergruppe $\boldsymbol{R}_{\boldsymbol{x}}{ }^{\prime}$ der Charaktergruppe $\boldsymbol{\Omega}_{\boldsymbol{x}}$ definiert werden. Daher ist die Faktorgruppe $\mathfrak{R}_{f} / \mathbb{R}_{r^{\prime}}{ }^{\prime}$ zu $\mathbb{R}^{\prime}$ isomorph. Damit ist also gezeigt:

Die Gruppe der Klassen ähnlicher Systeme, deren Faktorensysteme in $\boldsymbol{\Omega}_{r}$ liegen, ist zur Gruppe $\mathfrak{\Re}^{\prime}$ isomorph.

Zwei Faktorensysteme $c_{S, T}=\chi\left(C_{S, r}\right)$ und $c_{S, r}^{\prime}=\chi^{\prime}\left(C_{S, T}\right)$ sind dann und nur dann ähnlich, wenn $\chi^{\prime} \chi^{-1}(C)=1$ für jedes $C$ aus $\mathfrak{K}^{\prime}$ ist.

Es sei noch bemerkt, dass man die Sätze von Brauer über die Zerfällungskörper olhne weiteres auf unseren Fall übertragen kamn.

Mathematisches Institut

Kaiserliche Universität zu Osaka. 